\title{
Controller Design of Multiinput Multioutput Time-Delay Large-Scale System
}

\author{
Chia-Wei Lin, ${ }^{1}$ Tzuu-Hseng S. Li, ${ }^{1}$ and Chung-Cheng Chen ${ }^{2}$ \\ ${ }^{1}$ Department of Electrical Engineering, National Cheng Kung University, 1 University Road, Tainan 701, Taiwan \\ ${ }^{2}$ Department of Electrical Engineering, Hwa Hsia Institute of Technology, 111 Gong Jhuan Road, Chung Ho, Taipei 235, Taiwan \\ Correspondence should be addressed to Chung-Cheng Chen; ccc49827@ms25.hinet.net
}

Received 13 June 2013; Revised 28 July 2013; Accepted 28 July 2013

Academic Editor: Chang-Hua Lien

Copyright (c) 2013 Chia-Wei Lin et al. This is an open access article distributed under the Creative Commons Attribution License, which permits unrestricted use, distribution, and reproduction in any medium, provided the original work is properly cited.

\begin{abstract}
The paper presents a novel feedback linearization controller of nonlinear multiinput multioutput time-delay large-scale systems to obtain both the tracking and almost disturbance decoupling (ADD) performances. The significant contribution of this paper is to build up a control law such that the overall closed-loop system is stable for given initial condition and bounded tracking trajectory with the input-to-state-stability characteristic and almost disturbance decoupling performance. We have simulated the two-inverted-pendulum system coupled by a spring for networked control systems which has been used as a test bed for the study of decentralized control of large-scale systems.
\end{abstract}

\section{Introduction}

Recently, robust stabilization of system with time delay has been of a challenging and interesting problem [1]. As we know, in general, the existence of time-delay degrades the control performance and sometimes makes the closedloop stabilization difficult, especially for nonlinear systems. Appropriate mathematical descriptions incorporating the time delay are the differential-difference equations, that is, differential equations with deviating arguments. Several related reports have shown that differential-difference equations have been widely applied in theory of automatic control, the theory of self-oscillating systems, the study of problems connected with combustion in rocket motion, the problem of long-range planning in economics, a series of biological problems, and in many other areas of science and technology $[2,3]$. In the past, there have been a number of interesting developments in stability criteria and controller designs for time-delay control systems but mostly were restricted to linear cases; see, for example, [1, 4-6]. In general, the global stability test of control systems with time delays is not as easy, even in the linear case, as without time delays. It requires some disgusting tasks as solving nonlinear matrix equations [7]. It is clear that the investigation of nonlinear time-delay systems is worthwhile. In this paper, the globally tracking and almost disturbance decoupling problem of a general class of nonlinear time-delay control systems is investigated.

A large-scale system is organized as some interconnected subsystems, such as industrial control system, power systems, computer, biomedical networks, district heating systems [8], and wind farm power control [9]. Due to the inherited complexity of control approach and physical constraints on information communication between subsystems, it is necessary to design for each subsystem a decentralized controller depending on local data, even if to construct an object for the whole large-scale system. Recently networked control systems have attracted many research interests. The significant characteristics of networked control systems are controller decentralization, interconnected diagnostics, convenient maintenance, and low cost [10]. The application of networked control architecture will increase the performances of interconnected applications via reduced maintenance costs [11]. The major limitations in the existing networked control systems research are that (a) most researches are limited to linear cases and (b) the significant applications of networked control systems are for distributed control systems. However, some theoretical results are not valid for distributed control systems and then have little application value $[12,13]$. We 
have proposed a study of two-pendulum system coupled by a spring for networked control systems which has been used as a test bed for the investigation of decentralized control of large-scale systems.

Recently, variable control method has been utilized to investigate nonlinear industrial system. However, nasty chattering characteristics may excite unmodeled high frequency and even force system into instable state for variable control method [14]. The backstepping approach has been an important method for designing controller for nonlinear industrial systems. However, a disadvantage with the backstepping approach is the intricacy which is generated by the complex duplicated differentiations of some nonlinear functions [15]. The output regulation control method [16] is utilized to the industrial system in which the output terminals are derived with an exosystem. However, the output regulation problem should solve the complicated solution of partial-differential equation and the problem of creating the transient tracking errors. The nonlinear $H^{\infty}$ control method generally has to find out the solution for the complex Hamilton-Jacobi equation [17]. However, it is unfortunate that we can solve a closed-form solution only for some special industrial control systems [18]. The internal-model-principle method, solving a partial-differential center manifold equation [19], transforms the original tracking problem to output regulation structure. Only for some particular industrial systems and desired trajectories, the asymptotic solutions can be found out [20]. The $H^{\infty}$ adaptive fuzzy control approach has been utilized to systematically address some industrial control systems [1]. Its shortcoming is that the troublesome parameter update law makes the method impractical. During the past decade, the feedback linearization control has been the significant research direction for nonlinear systems [21] and has been addressed successfully to investigate many industrial control systems including the shunt hybrid power filter [22], high-power self-commutated voltage-source converter and current-source converter [23], and differential-drive wheeled mobile robots [24].

The almost disturbance decoupling control problem, originally developed for linear and nonlinear control systems by $[25,26]$, respectively, exploits the research that a controller reduces the effect of the disturbance on the outputs to an arbitrary degree of accuracy, and then many important researches have been proposed for nonlinear industrial control systems $[27,28]$. The authors of [26] exploit the fact that the almost disturbance decoupling performance cannot be achieved for the following control system: $\dot{x}_{1}(t)=\tan ^{-1}\left(x_{2}\right)+\theta(t), \dot{x}_{2}(t)=$ $u, y=x_{1}$, where $u, y$ are the input and output, respectively, and $\theta$ is the disturbance term. It is fortunate that this example can be easily solved via the proposed approach in this study. In order to exploit the significant industrial applicability, this study has favorably designed the almost disturbance decoupling controller for a two-inverted-pendulum system.

\section{Tracking and Almost Disturbance Decoupling Controller Design}

Consider the following nonlinear time-delay large-scale system with disturbance that is organized into $N$ subsystems interconnected by their output terminals. The $i$ th subsystem $S_{i}, 1 \leq i \leq N$, is shown as

$$
\begin{aligned}
\dot{x}_{i 1}= & f_{i 1}\left(x_{i 1}, x_{i 2}, \ldots, x_{i n_{i}}\right)+d_{i 1}\left(y_{i}\left(t-\tau_{i 1}(t)\right)\right) \\
& +\Delta f_{i 1}\left(y_{1}, y_{2}, \ldots, y_{N}\right)+\theta_{i 1} \\
\dot{x}_{i 2}= & f_{i 2}\left(x_{i 1}, x_{i 2}, \ldots, x_{i n_{i}}\right)+d_{i 2}\left(y_{i}\left(t-\tau_{i 2}(t)\right)\right) \\
& +\Delta f_{i 2}\left(y_{1}, y_{2}, \ldots, y_{N}\right)+\theta_{i 2} \\
& \vdots \\
& \quad \\
\dot{x}_{i n_{i}}= & f_{i n_{i}}\left(x_{i 1}, x_{i 2}, \ldots, x_{i n_{i}}\right)+d_{i n_{i}}\left(y_{i}\left(t-\tau_{i n_{i}}(t)\right)\right) \\
& +\Delta f_{i n_{i}}\left(y_{1}, y_{2}, \ldots, y_{N}\right)+\theta_{i n_{i}}+g_{i} u_{i}, \\
y_{i}= & x_{i 1},
\end{aligned}
$$

where $\left[\begin{array}{llll}x_{i 1}(t) & x_{i 2}(t) & \cdots & x_{i n_{i}}(t)\end{array}\right]^{T} \in \mathfrak{R}^{n_{i}}, u_{i n_{i}} \in \mathfrak{R}$, and $y_{i} \in \mathfrak{R}$ are the state vector, the input vector, and the output vector of subsystem $S_{i}$, respectively. $f_{i n_{i}}$ and $g_{i n_{i}}$ are smooth nonlinear system functions. $\Delta f_{i n_{i}}$ represents the nonlinearity in the $i$ th subsystem and the interconnection function between the $i$ th subsystem and other subsystems. $\theta_{i n_{i}}$ is a bounded time-varying disturbances vector, and $\tau_{i n_{i}}(t)$ is a time-delay term.

Define

$$
\begin{aligned}
N_{t} & \equiv \sum_{i=1}^{N} n_{i}, \\
x_{1} & \equiv x_{11}, x_{2} \equiv x_{12}, \ldots, x_{n_{1}} \equiv x_{1 n_{1}}, \\
x_{n_{1}+1} & \equiv x_{21}, x_{n_{1}+2} \equiv x_{22}, \ldots, x_{n_{1}+n_{2}} \equiv x_{2 n_{2}}, \\
& \vdots \\
x_{n_{1}+n_{2}+\cdots+n_{N-1}+1} & \equiv x_{N 1}, x_{n_{1}+n_{2}+\cdots+n_{N-1}+2} \\
& \equiv x_{N 2}, \ldots, x_{n_{1}+n_{2}+\cdots+n_{N}} \equiv x_{N n_{N}}, \\
n_{0} & \equiv 0, \\
x_{n_{0}+n_{1}+\cdots+n_{i}+j} & \equiv x_{(i+1)(j)}, \tau_{n_{0}+n_{1}+\cdots+n_{i}+j} \\
& \equiv \tau_{(i+1)(j)}, \Delta f_{n_{0}+n_{1}+\cdots+n_{i}+j} \\
D_{e\left(n_{0}+n_{1}+\cdots+n_{i}+j\right)} & \equiv \Delta f_{(i+1)(j)}, d_{n_{0}+n_{1}+\cdots+n_{i}+j} \equiv d_{(i+1)(j)(j)},
\end{aligned}
$$


The nonlinear time-delay large-scale system will be rewritten as

$$
\begin{aligned}
{\left[\begin{array}{c}
\dot{x}_{1} \\
\dot{x}_{2} \\
\vdots \\
\dot{x}_{N_{t}}
\end{array}\right]=} & {\left[\begin{array}{c}
f_{1}\left(x_{1}, x_{2}, \ldots, x_{N_{t}}\right) \\
f_{2}\left(x_{1}, x_{2}, \ldots, x_{N_{t}}\right) \\
\vdots \\
f_{N_{t}}\left(x_{1}, x_{2}, \ldots, x_{N_{t}}\right)
\end{array}\right]+\left[\begin{array}{c}
0_{\left(n_{1}-1\right) \times 1} \\
g_{1} u_{1} \\
0_{\left(n_{2}-1\right) \times 1} \\
g_{2} u_{2} \\
\vdots \\
0_{\left(n_{N}-1\right) \times 1} \\
g_{N} u_{N}
\end{array}\right] } \\
& +\left[\begin{array}{c}
D_{e 1}\left(\tau_{1}\right) \\
D_{e 2}\left(\tau_{2}\right) \\
\vdots \\
D_{e N_{t}}\left(\tau_{N_{t}}\right)
\end{array}\right]+\left[\begin{array}{c}
\Delta f_{1}\left(x_{1}, x_{2}, \ldots, x_{N_{t}}\right) \\
\Delta f_{2}\left(x_{1}, x_{2}, \ldots, x_{N_{t}}\right) \\
\vdots \\
\Delta f_{N_{t}}\left(x_{1}, x_{2}, \ldots, x_{N_{t}}\right)
\end{array}\right] \\
& +\sum_{j=1}^{N_{t}} q_{j}^{*} \theta_{j d}, \\
& {\left[\begin{array}{c}
y_{1} \\
y_{2} \\
y_{N}
\end{array}\right] \equiv\left[\begin{array}{c}
h_{1} \\
h_{2} \\
\vdots \\
h_{N}
\end{array}\right]=\left[\begin{array}{c}
x_{n_{1}+1} \\
\vdots \\
x_{n_{1}+n_{2}+\ldots+n_{N}+1}
\end{array}\right] . }
\end{aligned}
$$

That is,

$$
\begin{gathered}
\dot{X}(t)=f(X(t))+g u+D_{e}+\Delta f+\sum_{j=1}^{N_{t}} q_{j}^{*} \theta_{j d}, \\
y(t)=h(X(t)),
\end{gathered}
$$

where $X(t) \quad \equiv \quad\left[\begin{array}{llll}x_{1}(t) & x_{2}(t) & \cdots & x_{N_{t}}(t)\end{array}\right]^{T} \quad \epsilon$ $\mathfrak{R}^{N_{t}} \quad$ is the state vector, $g u$ 三 $\left[\begin{array}{lllllll}0_{\left(n_{1}-1\right) \times 1} & g_{1} u_{1} & 0_{\left(n_{2}-1\right) \times 1} & g_{2} u_{2} & \cdots & 0_{\left(n_{N}-1\right) \times 1} & g_{N} u_{N}\end{array}\right]^{T} \quad \epsilon$ $\mathfrak{R}^{N_{t}}$ is the input vector, $y \equiv\left[\begin{array}{llll}y_{1} & y_{2} & \cdots & y_{N}\end{array}\right]^{T} \in \mathfrak{R}^{N}$ is the output vector, $\theta_{d} \equiv\left[\begin{array}{llll}\theta_{1 d}(t) & \theta_{2 d}(t) & \cdots & \theta_{N_{t} d}(t)\end{array}\right]^{T} \in \mathfrak{R}^{N_{t}}$ is a bounded time-varying disturbances vector, and $\Delta f \equiv$ $\left[\begin{array}{llll}\Delta f_{1} & \Delta f_{2} & \cdots & \Delta f_{N_{t}}\end{array}\right] \in \mathfrak{R}^{N_{t}} \cdot f \equiv\left[\begin{array}{llll}f_{1} & f_{2} & \cdots & f_{N_{t}}\end{array}\right]^{T} \in \mathfrak{R}^{N_{t}}$, $D \equiv\left[\begin{array}{llll}D_{e 1} & D_{e 2} & \cdots & D_{e N_{t}}\end{array}\right]^{T} \in \mathfrak{R}^{N_{t}}, D_{e} \equiv \sum_{i=1}^{N_{t}} q_{i}^{*} D_{e i} \in$ $\mathfrak{R}^{N_{t}}$, and $h \equiv\left[\begin{array}{llll}h_{1} & h_{2} & \cdots & h_{N}\end{array}\right]^{T} \in \mathfrak{R}^{N}$ are smooth vector fields. The nominal system is then defined as follows:

$$
\begin{gathered}
\dot{X}(t)=f(X(t))+g u, \\
y(t)=h(X(t)) .
\end{gathered}
$$

The nominal system of the form (5a) and (5b) is said to have the vector relative degree $\left\{r_{1}, r_{2}, \ldots, r_{N}\right\}$ [29] with the following properties for all $X \in \mathfrak{R}^{N_{t}}$ :

(i)

$$
\begin{array}{r}
L_{g_{j}} L_{f}^{k} h_{i}(X)=0, \quad 1 \leq i \leq N, \\
1 \leq j \leq N, \quad k<r_{i}-1,
\end{array}
$$

where the operator $L$ is the Lie derivative [30] and $r_{1}+r_{2}+$ $\cdots+r_{N}=r$;

(ii) the $N \times N$ matrix

$$
A_{\text {non }} \equiv\left[\begin{array}{ccc}
L_{g_{1}} L_{f}^{r_{1}-1} h_{1}(X) & \cdots & L_{g_{N}} L_{f}^{r_{1}-1} h_{1}(X) \\
L_{g_{1}} L_{f}^{r_{2}-1} h_{2}(X) & \cdots & L_{g_{N}} L_{f}^{r_{2}-1} h_{2}(X) \\
\vdots & & \vdots \\
L_{g_{1}} L_{f}^{r_{N}-1} h_{N}(X) & & L_{g_{N}} L_{f}^{r_{N}-1} h_{N}(X)
\end{array}\right]
$$

is nonsingular.

The desired output trajectory $y_{d}^{i}, \quad 1 \leq i \leq N$ and its first $r_{i}$ derivatives are all uniformly bounded, and

$$
\left\|\left[y_{d}^{i}, y_{d}^{i(1)}, \ldots, y_{d}^{i\left(r_{i}\right)}\right]\right\| \leq B_{d}^{i}, \quad 1 \leq i \leq N
$$

where $B_{d}^{i}$ is some positive real constant. Based on the existence of vector relative degree, it has been shown [30] that the function

$$
\Omega: \mathfrak{R}^{N_{t}} \longrightarrow \mathfrak{R}^{N_{t}}
$$

defined as

$$
\begin{gathered}
\xi_{i} \equiv\left[\begin{array}{c}
\xi_{1}^{i} \\
\xi_{2}^{i} \\
\vdots \\
\xi_{r_{i}}^{i}
\end{array}\right] \equiv\left[\begin{array}{c}
\Omega_{1}^{i} \\
\Omega_{2}^{i} \\
\vdots \\
\Omega_{r_{i}}^{i}
\end{array}\right] \equiv\left[\begin{array}{c}
L_{f}^{0} h_{i}(X) \\
L_{f}^{1} h_{i}(X) \\
\vdots \\
L_{f}^{r_{i}-1} h_{i}(X)
\end{array}\right], \quad i=1,2, \ldots, N, \\
\Omega_{k}(X(t)) \equiv \eta_{k}(t), \quad k=r+1, r+2, \ldots, N_{t}, \\
L_{g_{j}} \Omega_{k}(X(t))=0, \quad k=r+1, r+2, \ldots, N_{t}, \quad 1 \leq j \leq N
\end{gathered}
$$

is a diffeomorphism, if (i) the distribution

$$
g_{\text {span }} \equiv \operatorname{span}\left\{g_{1}, g_{2}, \ldots, g_{N}\right\}
$$

has involutive property; (ii) the vector fields

$$
Z_{j}^{k}, \quad 1 \leq j \leq N, 1 \leq k \leq r_{j}
$$

are complete, where

$$
\begin{gathered}
Z_{j}^{k} \equiv(-1)^{k-1} a d_{\tilde{f}}^{k-1} \tilde{g}_{j}, \quad 1 \leq j \leq N, \quad 1 \leq k \leq r_{j}, \\
\tilde{f}(X) \equiv f(X)-g(X) A_{\mathrm{non}}^{-1}(X) s(X), \\
s(X) \equiv\left[\begin{array}{llll}
L_{f}^{r_{1}} h_{1}(X) & L_{f}^{r_{2}} h_{2}(X) & \cdots & L_{f}^{r_{N}} h_{N}(X)
\end{array}\right]^{T}, \\
\widetilde{g} \equiv\left[\begin{array}{llll}
\widetilde{g}_{1} & \widetilde{g}_{2} & \cdots & \widetilde{g}_{N}
\end{array}\right] \equiv g(X) A_{\mathrm{non}}^{-1}(X), \\
a d_{p}^{k} q \equiv\left[\begin{array}{lll}
p & a d_{p}^{k-1} q
\end{array}\right], \\
{\left[\begin{array}{ll}
p & q
\end{array}\right] \equiv \frac{\partial q}{\partial X} p(X)-\frac{\partial p}{\partial X} q(X) .}
\end{gathered}
$$


Denote the trajectory error to be

$$
\begin{aligned}
& e_{j}^{i} \equiv \xi_{j}^{i}-y_{d}^{i(j-1)}, \quad i=1,2, \ldots, N, j=1,2, \ldots, r_{i}, \\
& e^{i} \equiv\left[\begin{array}{llll}
e_{1}^{i} & e_{2}^{i} & \cdots & e_{r_{i}}^{i}
\end{array}\right]^{T} \in \mathfrak{R}^{r_{i}}, \\
& \overline{e_{j}^{i}} \equiv \kappa^{j-1} e_{j}^{i}, \quad i=1,2, \ldots, N, \quad j=1,2, \ldots, r_{i}, \\
& \overline{e^{i}} \equiv\left[\begin{array}{llll}
\overline{e_{1}^{i}} & \overline{e_{2}^{i}} & \cdots & \overline{e_{r_{i}}^{i}}(t)
\end{array}\right]^{T} \in \mathfrak{R}^{r_{i}}, \\
& \bar{e} \equiv\left[\begin{array}{llll}
\overline{e^{1}} & \overline{e^{2}} & \cdots & \overline{e^{N}}
\end{array}\right]^{T} \in \mathfrak{R}^{r}, \\
& \xi \equiv\left[\begin{array}{llll}
\xi_{1} & \xi_{2} & \cdots & \xi_{r}
\end{array}\right]^{T} \in \Re^{r}, \\
& \eta(t) \equiv\left[\begin{array}{llll}
\eta_{r+1}(t) & \eta_{r+2}(t) & \cdots & \eta_{N_{t}}(t)
\end{array}\right]^{T} \in \mathfrak{R}^{N_{t}-r}, \\
& q(\xi(t), \eta(t)) \equiv\left[\begin{array}{llll}
L_{f} \phi_{r+1}(t) & L_{f} \phi_{r+2}(t) & \cdots & L_{f} \phi_{N_{t}}(t)
\end{array}\right]^{T} \\
& \equiv\left[\begin{array}{llll}
q_{r+1} & q_{r+2} & \cdots & q_{N_{t}}
\end{array}\right]^{T} .
\end{aligned}
$$

Define a phase-variable canonical matrix $A_{\text {phase }}^{i}$ to be

$$
A_{\text {phase }}^{i} \equiv\left[\begin{array}{ccccc}
0 & 1 & 0 & \cdots & 0 \\
0 & 0 & 1 & \cdots & 0 \\
& \vdots & & & \vdots \\
0 & 0 & 0 & \cdots & 1 \\
-a_{1}^{i} & -a_{2}^{i} & -a_{3}^{i} & \cdots & -a_{r_{i}}^{i}
\end{array}\right]_{r_{i} \times r_{i}} \quad, \quad 1 \leq i \leq N
$$

where $a_{1}^{i}, a_{2}^{i}, \ldots, a_{r_{i}}^{i}$ are any chosen variable such that $A_{\text {phase }}^{i}$ is Hurwitz, and define the vector $B^{i}$ to be

$$
B^{i} \equiv\left[\begin{array}{lllll}
0 & 0 & \cdots & 0 & 1
\end{array}\right]_{r_{i} \times 1}^{T}, \quad 1 \leq i \leq N .
$$

Define the positive definite matrix $Q^{i}$ to be solution of the following Lyapunov equation:

$$
\left(A_{\text {phase }}^{i}\right)^{T} Q^{i}+Q^{i} A_{\text {phase }}^{i}=-I, \quad 1 \leq i \leq N,
$$

$\lambda_{\max }\left(Q^{i}\right) \equiv$ the maximum eigenvalue of $Q^{i}, \quad 1 \leq i \leq N$,

$\lambda_{\min }\left(Q^{i}\right) \equiv$ the minimum eigenvalue of $Q^{i}, \quad 1 \leq i \leq N$,

$$
\begin{aligned}
& \lambda_{\text {max }}^{*} \equiv \min \left\{\lambda_{\max }\left(Q^{1}\right), \lambda_{\max }\left(Q^{2}\right), \ldots, \lambda_{\max }\left(Q^{N}\right)\right\}, \\
& \lambda_{\text {min }}^{*} \equiv \min \left\{\lambda_{\min }\left(Q^{1}\right), \lambda_{\min }\left(Q^{2}\right), \ldots, \lambda_{\min }\left(Q^{N}\right)\right\} .
\end{aligned}
$$

Assumption 1. For all $t \geq 0, \eta \in \mathfrak{R}^{N_{t}-r}$, and $\xi \in \mathfrak{R}^{r}$, there exists a positive constant $L_{m}$ such that the following inequality holds:

$$
\left\|q_{22}(t, \eta, \bar{e})-q_{22}(t, \eta, 0)\right\| \leq L_{m}(\|\bar{e}\|)
$$

where $q_{22}(t, \eta, \bar{e}) \equiv q(\xi, \eta)$.
For the sake of describing precisely the considered problem, denote

$$
\begin{gathered}
d_{i j} \equiv L_{g_{j}} L_{f}^{r_{i}-1} h_{i}(X), \quad 1 \leq i \leq N, \quad 1 \leq j \leq N, \\
c_{i} \equiv L_{f}^{r_{i}} h_{i}(X), \quad 1 \leq i \leq N, \\
\overline{\overline{e^{i}}} \equiv a_{1}^{i} \overline{e_{1}^{i}}+a_{2}^{i} \overline{e_{2}^{i}}+\cdots+a_{r_{i}}^{i} \overline{e_{r_{i}}^{i}}, \quad 1 \leq i \leq N .
\end{gathered}
$$

Definition 2 (see [31]). Consider the nonlinear system $\dot{z}=$ $f(t, z, n)$, where $f:[0, \infty) \times \mathfrak{R}^{n} \times \mathfrak{R}^{n} \rightarrow \mathfrak{R}^{n}$ is piecewise continuous in $t$ and has the locally Lipschitz property in $z$ and $n$. This nonlinear system is said to has the input-to-state stable property if there exist a class $K L$ function $\beta$, a class $K$ function $\gamma$, and positive real constants $\lambda_{1}$ and $\lambda_{2}$ such that, for given initial state $z\left(t_{0}\right)$ with $\left\|z\left(t_{0}\right)\right\|<\lambda_{1}$ and any bounded noise $n(t)$ with $\sup _{t \geq t_{0}}\|n(t)\|<\lambda_{2}$, the system state has the following property:

$$
\|z(t)\| \leq \beta\left(\left\|z\left(t_{0}\right)\right\|, t-t_{0}\right)+\gamma\left(\sup _{t_{0} \leq \tau \leq t}\|n(\tau)\|\right)
$$

for all $t \geq t_{0} \geq 0$.

Definition 3 (see [32]). The tracking problem with almost disturbance decoupling performance is denoted to be globally solved by the control law $u$ for the overall system, if the control law $u$ has the following characteristics.

(i) The control system is input-to-state stable for disturbance inputs.

(ii) For any given initial value $\bar{x}_{e 0} \equiv\left[\begin{array}{ll}\bar{e}\left(t_{0}\right) & \eta\left(t_{0}\right)\end{array}\right]^{T}$, for any $t \geq t_{0}$, and for any $t_{0} \geq 0$,

$$
\begin{aligned}
\left|y(t)-y_{d}(t)\right| \leq & \beta_{11}\left(\left\|x\left(t_{0}\right)\right\|, t-t_{0}\right) \\
& +\frac{1}{\sqrt{\beta_{22}}} \beta_{33}\left(\sup _{t_{0} \leq \tau \leq t}\|\theta(\tau)\|\right), \\
& \int_{t_{0}}^{t}\left[y(\tau)-y_{d}(\tau)\right]^{2} d \tau \\
& \leq \frac{1}{\beta_{44}}\left[\beta_{55}\left(\left\|\bar{x}_{e 0}\right\|\right)+\int_{t_{0}}^{t} \beta_{33}\left(\|\theta(\tau)\|^{2}\right) d \tau\right],
\end{aligned}
$$

where $\beta_{22}, \beta_{44}$ are positive real constants, $\beta_{33}, \beta_{55}$ are class $K$ functions, and $\beta_{11}$ is a class $K L$ function.

Theorem 4. Assume that there exists a continuously differentiable function $T: \mathfrak{R}^{N_{t^{-}}} \rightarrow \mathfrak{R}^{+}$such that the following properties hold for all $\eta \in \mathfrak{R}^{N_{t}-r}$ :

$$
\begin{gathered}
\Delta_{1}\|\eta\|^{2} \leq T(\eta) \leq \Delta_{2}\|\eta\|^{2}, \quad \Delta_{1}, \Delta_{2}>0, \\
\nabla_{t} T+\left(\nabla_{\eta} T\right)^{T} q_{22}(t, \eta, 0) \leq-2 \alpha_{x}\|\eta\|^{2}, \quad \alpha_{x}>0, \\
\left\|\nabla_{\eta} T\right\| \leq \Delta_{3}\|\eta\|, \quad \Delta_{3}>0 .
\end{gathered}
$$


Then the tracking problem with almost disturbance decoupling performance for time-delay large-scale system is globally solved by the control law:

$$
\begin{gathered}
u=A_{\text {non }}^{-1}\left\{-u_{b}+u_{v}\right\} \\
u_{b} \equiv\left[\begin{array}{llll}
L_{f}^{r_{1}} h_{1} & L_{f}^{r_{2}} h_{2} & \cdots & L_{f}^{r_{N}} h_{N}
\end{array}\right]^{T}, \\
u_{v} \equiv\left[\begin{array}{llll}
v_{1} & v_{2} & \cdots & v_{N}
\end{array}\right]^{T}, \\
v_{i} \equiv y_{d}^{i\left(r_{i}\right)}-\kappa^{-r_{i}} a_{1}^{i}\left[\begin{array}{ll}
L_{f}^{0} h_{i}(X)-y_{d}^{i}
\end{array}\right] \\
-\kappa^{1-r_{i}} a_{2}^{i}\left[L_{f}^{1} h_{i}(X)-y_{d}^{i(1)}\right]
\end{gathered}
$$

Moreover, the influence of disturbances on the $L_{2}$ norm of the tracking error can be arbitrarily reduced by adjusting parameter $\mathrm{N}_{2}>1$ :

$k_{11} \equiv \frac{k}{2 \kappa}-\frac{25 k^{2}}{\kappa^{2}}\left\|\phi_{\xi}^{1}\right\|^{2}\left\|Q^{1}\right\|^{2}-\cdots-\frac{25 k^{2}}{\kappa^{2}}\left\|\phi_{\xi}^{N}\right\|^{2}\left\|Q^{N}\right\|^{2}-25$,

$$
k_{22} \equiv 22 \alpha_{x}-\frac{1}{100} \Delta_{3}^{2} L_{m}^{2}-25 \Delta_{3}^{2}\left\|\phi_{\eta}\right\|^{2}
$$

$$
N_{2} \equiv \min \left\{k_{11}, k_{22}\right\}
$$

$$
\Omega_{\xi}^{i}(\kappa) \equiv\left[\begin{array}{ccc}
\kappa \frac{\partial}{\partial X} h_{i} q_{1}^{*} & \cdots & \kappa \frac{\partial}{\partial X} h_{i} q_{p}^{*} \\
\vdots & & \vdots \\
\kappa^{r_{i}} \frac{\partial}{\partial X} L_{f}^{r_{i}-1} h_{i} q_{1}^{*} & \cdots & \kappa^{r_{i}} \frac{\partial}{\partial X} L_{f}^{r_{i}-1} h_{i} q_{q}^{*}
\end{array}\right]
$$

$$
1 \leq i \leq N
$$

$$
\Omega_{\eta}(\kappa) \equiv\left[\begin{array}{ccc}
\frac{\partial}{\partial X} \phi_{r+1} q_{1}^{*} & \cdots & \frac{\partial}{\partial X} \phi_{r+1} q_{p}^{*} \\
\vdots & & \vdots \\
\frac{\partial}{\partial X} \phi_{N_{t}} q_{1}^{*} & \cdots & \frac{\partial}{\partial X} \phi_{N_{t}} q_{q}^{*}
\end{array}\right]
$$

where $k(\kappa): \mathfrak{R}^{+} \rightarrow \mathfrak{R}^{+}$is a continuous function satisfying

$$
\lim _{\varepsilon \rightarrow 0} k(\kappa)=0, \quad \lim _{\kappa \rightarrow 0} \frac{\kappa}{k(\kappa)}=0 .
$$

Proof. Applying the coordinate transformation (9) yields

$$
\begin{aligned}
\dot{\xi}_{1}^{1}= & \frac{\partial h_{1}}{\partial X} f+\sum_{j=1}^{N_{t}} \frac{\partial h_{1}}{\partial X} q_{j}^{*}\left(\theta_{j d}+D_{e j}\right) \\
= & \xi_{2}^{1}+\sum_{j=1}^{N_{t}} \frac{\partial h_{1}}{\partial X} q_{j}^{*}\left(\theta_{j d}+D_{e j}\right), \\
& \vdots \\
\dot{\xi}_{r_{1}-1}^{1}= & \frac{\partial L_{f}^{r_{1}-2} h_{1}}{\partial X} f+\sum_{j=1}^{N_{t}} \frac{\partial L_{f}^{r_{1}-2} h_{1}}{\partial X} q_{j}^{*}\left(\theta_{j d}+D_{e j}\right) \\
= & L_{f}^{r_{1}-1} h_{1}+\sum_{j=1}^{N_{t}} \frac{\partial L_{f}^{r_{1}-2} h_{1}}{\partial X} q_{j}^{*}\left(\theta_{j d}+D_{e j}\right), \\
\dot{\xi}_{r_{1}}^{1}= & c_{1}+d_{11} u_{1}+\cdots+d_{1 N} u_{N} \\
& +\sum_{j=1}^{N_{t}} \frac{\partial L_{f}^{r_{1}-1} h_{1}}{\partial X} q_{j}^{*}\left(\theta_{j d}+D_{e j}\right),
\end{aligned}
$$

$$
\begin{aligned}
\dot{\xi}_{1}^{N}= & L_{f}^{1} h_{N}+\sum_{j=1}^{N_{t}} \frac{\partial h_{1}}{\partial X} q_{j}^{*}\left(\theta_{j d}+D_{e j}\right) \\
= & \xi_{2}^{N}+\sum_{j=1}^{N_{t}} \frac{\partial h_{N}}{\partial X} q_{j}^{*}\left(\theta_{j d}+D_{e j}\right), \\
& \vdots \\
\dot{\xi}_{r_{N}-1}^{N}= & L_{f}^{r_{N}-1} h_{N}+\sum_{j=1}^{N_{t}} \frac{\partial L_{f}^{r_{N}-2} h_{N}}{\partial X} q_{j}^{*}\left(\theta_{j d}+D_{e j}\right) \\
= & \xi_{r_{N}}^{N}+\sum_{j=1}^{N_{t}} \frac{\partial L_{f}^{r_{N}-2} h_{N}}{\partial X} q_{j}^{*}\left(\theta_{j d}+D_{e j}\right), \\
\dot{\xi}_{r_{N}}^{N}= & c_{N}+d_{N 1} u_{1}+\cdots+d_{N N} u_{N} \\
\dot{\eta}_{k}(t)= & L_{f} \Omega_{k}+\sum_{j=1}^{N_{t}} \frac{\partial \Omega_{k}}{\partial X} q_{j}^{*}\left(\theta_{j d}+D_{e j}\right) \\
& +\sum_{j=1}^{N_{t}} \frac{\partial L_{f}^{r_{N}-1} h_{N}}{\partial X} q_{j}^{*}\left(\theta_{j d}+D_{e j}\right), \\
& q_{k}+\sum_{j=1}^{N_{t}} \frac{\partial \Omega_{k}}{\partial X} q_{j}^{*}\left(\theta_{j d}+D_{e j}\right) . \\
& \\
&
\end{aligned}
$$


Since

$$
\begin{gathered}
c_{i}(\xi(t), \eta(t)) \equiv L_{f}^{r_{i}} h_{i}(X(t)), \quad 1 \leq i \leq N, \\
d_{i j} \equiv L_{g_{j}} L_{f}^{r_{i}-1} h_{i}(X), \quad 1 \leq i \leq N, \quad 1 \leq j \leq N, \\
q_{k}(\xi(t), \eta(t))=L_{f} \Omega_{k}(X), \quad k=r+1, r+2, \ldots, N_{t} .
\end{gathered}
$$

The dynamic equations of systems (3a) and (3b) can be rewritten as follows:

$$
\begin{aligned}
& \dot{\xi}_{i}^{1}(t)=\xi_{i+1}^{1}(t)+\sum_{j=1}^{N_{t}} \frac{\partial}{\partial X} L_{f}^{i-1} h_{1} q_{j}^{*}\left(\theta_{j d}+D_{e j}\right), \\
& i=1,2, \ldots, r_{1}-1 \text {, } \\
& \dot{\xi}_{r_{1}}^{1}(t)=c_{1}(\xi(t), \eta(t))+d_{11}(\xi(t), \eta(t)) u_{1} \\
& +\cdots+d_{1 N}(\xi(t), \eta(t)) u_{N} \\
& +\sum_{j=1}^{N_{t}} \frac{\partial}{\partial X} L_{f}^{r_{1}-1} h_{1} q_{j}^{*}\left(\theta_{j d}+D_{e j}\right) \\
& \dot{\xi}_{i}^{N}(t)=\xi_{i+1}^{N}(t)+\sum_{j=1}^{N_{t}} \frac{\partial}{\partial X} L_{f}^{i-1} h_{N} q_{j}^{*}\left(\theta_{j d}+D_{e j}\right), \\
& i=1,2, \ldots, r_{N}-1 \text {, } \\
& \dot{\xi}_{r_{N}}^{N}(t)=c_{N}(\xi(t), \eta(t))+d_{N 1}(\xi(t), \eta(t)) u_{1} \\
& +\cdots+d_{N N}(\xi(t), \eta(t)) u_{N} \\
& +\sum_{j=1}^{N_{t}} \frac{\partial}{\partial X} L_{f}^{r_{N}-1} h_{N} q_{j}^{*}\left(\theta_{j d}+D_{e j}\right) \\
& \dot{\eta}_{k}(t)=q_{k}(\xi(t), \eta(t))+\sum_{j=1}^{N_{t}} \frac{\partial}{\partial X} \Omega_{k}(X) q_{j}^{*}\left(\theta_{j d}+D_{e j}\right), \\
& k=r+1, \ldots, N_{t} \text {, } \\
& y_{i}(t)=\xi_{1}^{i}(t), \quad 1 \leq i \leq N .
\end{aligned}
$$

Applying (14), (34), (37), and (38) yields the controller as

$$
u=A_{\text {non }}^{-1}\left[-u_{b}+u_{v}\right]
$$

The dynamic equations of systems (3a) and (3b) can be rewritten as follows by substituting (46) into (41) and (43):

$\left[\begin{array}{c}\dot{\xi}_{1}^{i}(t) \\ \dot{\xi}_{2}^{i}(t) \\ \vdots \\ \dot{\xi}_{r_{i}-1}^{i}(t) \\ \dot{\xi}_{r_{i}}^{i}(t)\end{array}\right]=\left[\begin{array}{cccccc}0 & 1 & 0 & & \cdots & 0 \\ 0 & 0 & 1 & 0 & \cdots & 0 \\ & \vdots & & & \vdots \\ 0 & 0 & 0 & \cdots & 1 \\ 0 & 0 & 0 & & \cdots & 0\end{array}\right]\left[\begin{array}{c}\xi_{1}^{i}(t) \\ \xi_{2}^{i}(t) \\ \vdots \\ \xi_{r_{i}-1}^{i}(t) \\ \xi_{r_{i}}^{i}(t)\end{array}\right]$

$$
+\left[\begin{array}{c}
0 \\
0 \\
\vdots \\
0 \\
1
\end{array}\right] v_{i}+\left[\begin{array}{c}
\sum_{j=1}^{N_{t}} \frac{\partial}{\partial X} h_{i} q_{j}^{*}\left(\theta_{j d}+D_{e j}\right) \\
\sum_{j=1}^{N_{t}} \frac{\partial}{\partial X} L_{f}^{1} h_{i} q_{j}^{*}\left(\theta_{j d}+D_{e j}\right) \\
\vdots \\
\sum_{j=1}^{N_{t}} \frac{\partial}{\partial X} L_{f}^{r_{i}-1} h_{i} q_{j}^{*}\left(\theta_{j d}+D_{e j}\right)
\end{array}\right],
$$$$
\left[\begin{array}{c}
\dot{\eta}_{r+1}(t) \\
\dot{\eta}_{r+2}(t) \\
\vdots \\
\dot{\eta}_{N_{t}-1}(t) \\
\dot{\eta}_{N_{t}}(t)
\end{array}\right]=\left[\begin{array}{c}
q_{r+1}(t) \\
q_{r+2}(t) \\
\vdots \\
q_{N_{t}-1}(t) \\
q_{N_{t}}(t)
\end{array}\right]+\left[\begin{array}{c}
\sum_{j=1}^{N_{t}} \frac{\partial}{\partial X} \Omega_{r+1} q_{j}^{*}\left(\theta_{j d}+D_{e j}\right) \\
\sum_{j=1}^{N_{t}} \frac{\partial}{\partial X} \Omega_{r+2} q_{j}^{*}\left(\theta_{j d}+D_{e j}\right) \\
\vdots \\
\sum_{j=1}^{N_{t}} \frac{\partial}{\partial X} \Omega_{N_{t}-1} q_{j}^{*}\left(\theta_{j d}+D_{e j}\right) \\
\sum_{j=1}^{N_{t}} \frac{\partial}{\partial X} \Omega_{N_{t}} q_{j}^{*}\left(\theta_{j d}+D_{e j}\right)
\end{array}\right],
$$$$
y_{i}=\left[\begin{array}{lllll}
1 & 0 & \cdots & 0 & 0
\end{array}\right]_{r \times 1}\left[\begin{array}{c}
\xi_{1}^{i}(t) \\
\xi_{2}^{i}(t) \\
\vdots \\
\xi_{r_{i}-1}^{i}(t) \\
\xi_{r_{i}}^{i}(t)
\end{array}\right]_{r \times 1}
$$

$$
=\xi_{1}^{i}(t), \quad 1 \leq i \leq N
$$

Combining (14), (16), (17), (22), and (34) yields the transformation of (47):

$$
\begin{array}{r}
\dot{\eta}(t)=q(\xi(t), \eta(t))+\Omega_{\eta}\left(\theta_{d}+D\right) \\
\equiv q_{22}(t, \eta(t), \bar{e})+\Omega_{\eta}\left(\theta_{d}+D\right), \\
\dot{\kappa \bar{e}^{i}}(t)=A_{\text {phase }}^{i} \overline{e^{i}}+\Omega_{\xi}^{i}\left(\theta_{d}+D\right), \quad 1 \leq i \leq N,
\end{array}
$$

$$
y_{i}(t)=\xi_{1}^{i}(t), \quad 1 \leq i \leq N .
$$


We consider $L(\bar{e}, \eta)$ denoted by a weighted sum of $T(\eta)$ and $W(\bar{e})$, and

$$
\begin{aligned}
L(\bar{e}, \eta) & \equiv T(\eta)+k(\kappa) W(\bar{e}) \\
& \equiv T(\eta)+k(\kappa)\left(W^{1}\left(\overline{e^{1}}\right)+\cdots+W^{N}\left(\overline{e^{N}}\right)\right),
\end{aligned}
$$

where

$$
W(\bar{e}) \equiv W^{1}\left(\overline{e^{1}}\right)+\cdots+W^{N}\left(\overline{e^{N}}\right)
$$

as a composite Lyapunov function of the subsystems (48a) and $(48 \mathrm{~b})[33,34]$, where $W\left(\overline{e^{i}}\right)$ satisfies

$$
W^{i}\left(\overline{e^{i}}\right) \equiv \frac{1}{2}{\overline{e^{i}}}^{T} Q^{i} \overline{e^{i}}
$$

Utilizing (14), (25) and (30a), (30b), and (30c) result in the derivative of $L$ along the trajectories of (48a) and (48b) as

$$
\begin{aligned}
\dot{L}= & {\left[\nabla_{t} T+\left(\nabla_{\eta} T\right)^{T} \dot{\eta}\right] } \\
& +\frac{k}{2}\left[\left(\overline{e^{1}}\right)^{T} Q^{1} \overline{e^{1}}+\left(\overline{e^{1}}\right)^{T} Q^{1}\left(\overline{e^{1}}\right)\right. \\
& \left.+\cdots+\left(\overline{e^{N}}\right)^{T} Q^{N} \overline{e^{N}}+\left(\overline{e^{N}}\right)^{T} Q^{N}\left(\overline{e^{N}}\right)\right] \\
\leq & {\left[\nabla_{t} T+\left(\nabla_{\eta} T\right)^{T} q_{22}(t, \eta(t), \bar{e})+\left(\nabla_{\eta} V\right)^{T} \Omega_{\eta}\left(\theta_{d}+D\right)\right] } \\
& -\frac{k}{2 \kappa}\left[\left(\overline{e^{1}}\right)^{T} \overline{e^{1}}+\cdots+\left(\overline{e^{N}}\right)^{T} \overline{e^{N}}\right] \\
& +\frac{k}{\kappa}\left[\left\|\left(\theta_{d}+D\right)\right\|\left\|\Omega_{\xi}^{1}\right\|\left\|Q^{1}\right\|\left\|\overline{e^{1}}\right\|\right. \\
& +\frac{N+1}{100}\left\|\left(\theta_{d}+D\right)\right\|^{2} . \\
\leq & -\|\eta\|^{2}\left[2 \alpha_{x}-\frac{1}{100} \Delta_{3}^{2} L_{m}^{2}-25 \Delta_{3}^{2}\left\|\Omega_{\eta}\right\|^{2}\right] \\
- & \|\bar{e}\|^{2}\left[\frac{k}{2 \kappa}-\frac{25 k^{2}}{\kappa^{2}}\left\|\Omega_{\xi}^{1}\right\|^{2}\left\|Q^{1}\right\|^{2}\right. \\
& \left.+\cdots\left(\theta_{d}+D\right)\|\| \Omega_{\xi}^{N}\|\| Q^{N}\|\| \overline{e^{N}} \|\right]
\end{aligned}
$$

That is,

$$
\dot{L} \leq-k_{11}\|\bar{e}\|^{2}-k_{22}\|\eta\|^{2}+\frac{N+1}{100}\left\|\left(\theta_{d}+D\right)\right\|^{2} .
$$

From (35c), we obtain

$$
\dot{L} \leq-N_{2}\left(\|\bar{e}\|^{2}+\|\eta\|^{2}\right)+\frac{N+1}{100}\left\|\left(\theta_{d}+D\right)\right\|^{2} .
$$

Define

$$
\bar{e} \equiv\left[\begin{array}{c}
\overline{e^{1}} \\
\overline{e^{2}} \\
\vdots \\
\overline{e^{N}}
\end{array}\right] \equiv\left[\begin{array}{c}
\overline{e_{1}^{1}} \\
\overline{e_{\mathrm{rem}}^{1}}
\end{array}\right], \quad \overline{e_{\mathrm{rem}}^{1}} \in \mathfrak{R}^{r-1}
$$

Hence

$$
\dot{L} \leq-N_{2}\left(\|\eta\|^{2}+\left\|\overline{e_{1}^{1}}\right\|^{2}+\left\|\overline{e_{\text {rem }}^{1}}\right\|^{2}\right)+\frac{N+1}{100}\left\|\left(\theta_{d}+D\right)\right\|^{2} .
$$

Utilizing (57) easily yields

$$
\begin{aligned}
& \int_{t_{0}}^{t}\left(y_{1}(\tau)-y_{d}^{1}(\tau)\right)^{2} d \tau \\
& \quad \leq \frac{L\left(t_{0}\right)}{N_{2}}+\frac{N+1}{100 N_{2}} \int_{t_{0}}^{t}\left\|\left(\theta_{d}(\tau)+D\right)\right\|^{2} d \tau .
\end{aligned}
$$

Similarly, it is easy to prove that

$$
\begin{aligned}
& \int_{t_{0}}^{t}\left(y_{i}(\tau)-y_{d}^{i}(\tau)\right)^{2} d \tau \\
& \leq \frac{L\left(t_{0}\right)}{N_{2}}+\frac{N+1}{100 N_{2}} \int_{t_{0}}^{t}\left\|\left(\theta_{d}(\tau)+D\right)\right\|^{2} d \tau, \\
& \quad 2 \leq i \leq N
\end{aligned}
$$

so that statement (29) is satisfied. From (55), we get

$$
\dot{L} \leq-N_{2}\left(\left\|y_{\text {total }}\right\|^{2}\right)+\frac{N+1}{100}\left\|\left(\theta_{d}+D\right)\right\|^{2},
$$

where

$$
\left\|y_{\text {total }}\right\|^{2} \equiv\|\bar{e}\|^{2}+\|\eta\|^{2}
$$

Utilizing [31, Theorem 5.2] and (60a) implies the input-tostate stable property for the overall system. Furthermore, it is easy to obtain the following inequality:

$$
\Delta_{\min }\left(\|\bar{e}\|^{2}+\|\eta\|^{2}\right) \leq L \leq \Delta_{\max }\left(\|\bar{e}\|^{2}+\|\eta\|^{2}\right) .
$$

That is,

$$
\Delta_{\min }\left(\left\|y_{\text {total }}\right\|^{2}\right) \leq L \leq \Delta_{\max }\left(\left\|y_{\text {total }}\right\|^{2}\right),
$$

where $\Delta_{\min } \equiv \min \left\{\Delta_{1},(k / 2) \lambda_{\min }^{*}\right\}$ and $\Delta_{\max } \equiv$ $\max \left\{\Delta_{2},(k / 2) \lambda_{\text {max }}^{*}\right\}$. From (55) and (62), we get

$$
\dot{L} \leq-\frac{N_{2}}{\Delta_{\max }} L+\frac{N+1}{100}\left(\sup _{t_{0} \leq \tau \leq t}\left\|\left(\theta_{d}(\tau)+D\right)\right\|\right)^{2} .
$$

Hence,

$$
\begin{aligned}
L(t) \leq & L\left(t_{0}\right) e^{\left(-N_{2} / \Delta_{\max }\right)\left(t-t_{0}\right)} \\
& +\frac{\Delta_{\max }(N+1)}{100 N_{2}}\left(\sup _{t_{0} \leq \tau \leq t}\left\|\left(\theta_{d}(\tau)+D\right)\right\|\right)^{2},
\end{aligned}
$$


which implies

$$
\begin{aligned}
\left|y_{1}(t)-y_{d}^{1}(t)\right| \leq & \sqrt{\frac{2 L\left(t_{0}\right)}{k \lambda_{\min }^{*}}} e^{\left(-N_{2} / 2 \Delta_{\max }\right)\left(t-t_{0}\right)} \\
& +\sqrt{\frac{\Delta_{\max }(N+1)}{50 k \lambda_{\min }^{*} N_{2}}}\left(\sup _{t_{0} \leq \tau \leq t}\left\|\left(\theta_{d}(\tau)+D\right)\right\|\right) .
\end{aligned}
$$

Similarly, it is easy to prove that

$$
\begin{aligned}
& \left|y_{i}(t)-y_{d}^{1}(t)\right| \\
& \leq \sqrt{\frac{2 L\left(t_{0}\right)}{k \lambda_{\min }^{*}}} e^{\left(-N_{2} / 2 \Delta_{\max }\right)\left(t-t_{0}\right)}+\sqrt{\frac{\Delta_{\max }(N+1)}{50 k \lambda_{\min }^{*} N_{2}}} \\
& \quad \times\left(\sup _{t_{0} \leq \tau \leq t}\left\|\left(\theta_{d}(\tau)+D\right)\right\|\right), \quad 2 \leq i \leq N
\end{aligned}
$$

so that statement (28) is proved, and then the tracking problem with almost disturbance decoupling is globally solved.

If the sum $r_{1}+r_{2}+\cdots+r_{m}$ is equal to the system dimension $n$, then Theorem 4 will be reduced to the following simplified version with cancelling Assumption 1 and (30a), (30b), and (30c).

Theorem 5. The tracking problem with almost disturbance decoupling performance for time-delay large-scale system is globally solved by the control law:

$$
\begin{gathered}
u=A_{n o n}^{-1}\left\{-u_{b}+u_{v}\right\}, \\
u_{b} \equiv\left[\begin{array}{llll}
L_{f}^{r_{1}} h_{1} & L_{f}^{r_{2}} h_{2} & \cdots & L_{f}^{r_{N}} h_{N}
\end{array}\right]^{T}, \\
u_{v} \equiv\left[\begin{array}{llll}
v_{1} & v_{2} & \cdots & v_{N}
\end{array}\right]^{T}, \\
v_{i} \equiv y_{d}^{i\left(r_{i}\right)}-\kappa^{-r_{i}} a_{1}^{i}\left[\begin{array}{ll}
L_{f}^{0} h_{i}(X)-y_{d}^{i}
\end{array}\right] \\
-\kappa^{1-r_{i}} a_{2}^{i}\left[L_{f}^{1} h_{i}(X)-y_{d}^{i(1)}\right] \\
-\cdots-\kappa^{-1} a_{r_{i}}^{i}\left[L_{f}^{r_{i}-1} h_{i}(X)-y_{d}^{i\left(r_{i}-1\right)}\right], \quad 1 \leq i \leq N .
\end{gathered}
$$

Moreover, the influence of disturbances on the $L_{2}$ norm of the tracking error can be arbitrarily reduced by adjusting parameter $k_{11}>1$ :

$$
k_{11} \equiv \frac{k}{2 \kappa}-\frac{25 k^{2}}{\kappa^{2}}\left\|\phi_{\xi}^{1}\right\|^{2}\left\|Q^{1}\right\|^{2}-\cdots-\frac{25 k^{2}}{\kappa^{2}}\left\|\phi_{\xi}^{N}\right\|^{2}\left\|Q^{N}\right\|^{2} .
$$

\section{Illustrative Example}

Consider the two inverted pendulums coupled by a spring with disturbances as shown in Figure 1. The dynamic equations are given as follows:

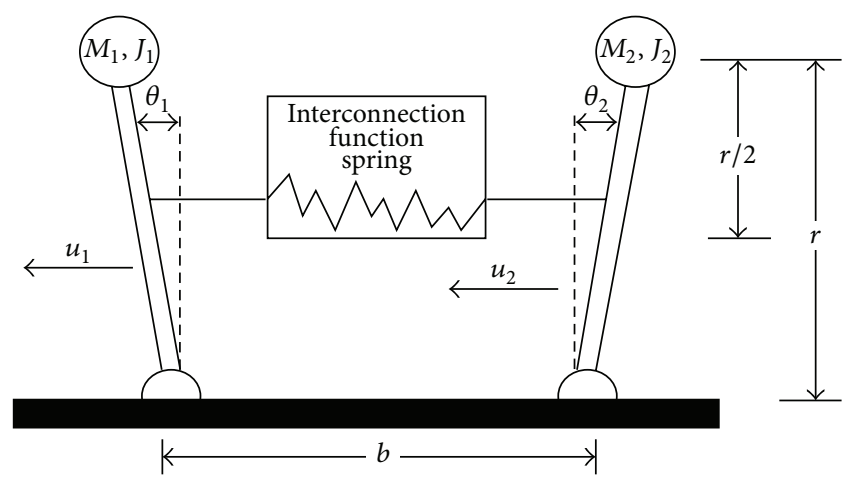

FIGURE 1: The two-inverted-pendulum system coupled by a spring.

$S_{1}$ (inverted pendulum 1):

$$
\begin{gathered}
\ddot{\theta}_{1}(t)=\left(-\frac{k r^{2}}{4 J_{1}}+\frac{m_{1} g r}{J_{1}}\right) \sin \left(\theta_{1}(t)\right) \\
+\frac{k r(l-b)}{2 J_{1}}+\frac{k r^{2}}{4 J_{1}} \sin \left(\theta_{2}(t)\right) \\
+\frac{u_{1}}{J_{1}}+\frac{\theta_{1}\left(t-\tau_{1,2}(t)\right)}{1+\theta_{1}^{2}\left(t-\tau_{1,2}(t)\right)} \\
\tau_{1,2}(t) \equiv 0.4\left(1+\sin ^{2}(t)\right), \\
y_{1}=\theta_{1}(t),
\end{gathered}
$$

$S_{2}$ (inverted pendulum 2):

$$
\begin{gathered}
\ddot{\theta}_{2}(t)=\left(-\frac{k r^{2}}{4 J_{2}}+\frac{m_{2} g r}{J_{2}}\right) \sin \left(\theta_{2}(t)\right) \\
+\frac{k r(l-b)}{2 J_{2}}+\frac{k r^{2}}{4 J_{2}} \sin \left(\dot{\theta}_{1}(t)\right) \\
+\frac{u_{2}}{J_{2}}+\frac{\theta_{2}\left(t-\tau_{2,2}(t)\right)}{1+\theta_{2}^{2}\left(t-\tau_{2,2}(t)\right)} \\
\tau_{2,2}(t) \equiv 0.4\left(1+\sin ^{2}(t)\right) \\
y_{2}=\theta_{2}(t),
\end{gathered}
$$

where $u_{i}$ is the torque input generated by the actuator for pendulum $i(i=1,2), \theta_{i}$ is the angular displacement of pendulum $i(i=1,2), m_{i}$ is the mass of pendulum $i(i=1,2)$, $J_{i}$ is the moment of inertia $(i=1,2), k$ is the spring constant, $r$ is the pendulum height, $l$ is the length of pendulum, $g$ is the gravitational acceleration constant, and $b$ is the distance between the pendulums. Defining the state variables 
$x_{1} \equiv \theta_{1}(t), x_{2} \equiv \dot{\theta}_{1}(t), x_{3} \equiv \theta_{2}(t)$, and $x_{4} \equiv \dot{\theta}_{2}(t)$ yields the following state space models:

$$
\begin{gathered}
\dot{x}_{1}(t)=x_{2}(t), \\
\dot{x}_{2}(t)=\left(-\frac{k r^{2}}{4 J_{1}}+\frac{m_{1} g r}{J_{1}}\right) \sin \left(x_{1}(t)\right) \\
+\frac{k r(l-b)}{2 J_{1}}+\frac{k r^{2}}{4 J_{1}} \sin \left(x_{3}(t)\right) \\
+\frac{u_{1}}{J_{1}}+\frac{x_{1}\left(t-\tau_{1,2}(t)\right)}{1+x_{1}^{2}\left(t-\tau_{1,2}(t)\right)}, \\
\dot{x}_{4}(t)=\left(-\frac{\dot{x}_{3}(t)=x_{4}(t),}{4 J_{1}}+\frac{m_{1} g r}{J_{1}}\right) \sin \left(x_{3}(t)\right) \\
+\frac{k r(l-b)}{2 J_{1}}+\frac{k r^{2}}{4 J_{1}} \sin \left(x_{2}(t)\right) \\
+\frac{u_{2}}{J_{2}}+\frac{x_{3}\left(t-\tau_{1,2}(t)\right)}{1+x_{3}^{2}\left(t-\tau_{1,2}(t)\right)} \\
\tau_{1,2}(t) \equiv \tau_{2,2}(t) \equiv 0.4\left(1+\sin ^{2}(t)\right), \\
y_{1}=x_{1}(t), \\
y_{2}=x_{3}(t) .
\end{gathered}
$$

The following physical parameters are chosen in our simulation: $m_{1}=2 \mathrm{~kg}, m_{2}=2.5 \mathrm{~kg}, J_{1}=1 \mathrm{~kg} / \mathrm{m}^{2}, J_{2}=$ $1 \mathrm{~kg} / \mathrm{m}^{2}, k=10 \mathrm{~N} / \mathrm{m}, r=0.1 \mathrm{~m}, l=0.5 \mathrm{~m}, g=9.8 \mathrm{~m} / \mathrm{s}^{2}$, and $b=0.5 \mathrm{~m}$. Hence the mathematical model can be rewritten as

$$
\begin{gathered}
\dot{x}_{1}(t)=x_{2}(t), \\
\dot{x}_{2}(t)=1.935 \sin \left(x_{1}(t)\right)+0.025 \sin \left(x_{3}(t)\right) \\
+u_{1}+\frac{x_{1}\left(t-0.4\left(1+\sin ^{2}(t)\right)\right)}{1+x_{1}^{2}\left(t-0.4\left(1+\sin ^{2}(t)\right)\right)} \\
\dot{x}_{3}(t)=x_{4}(t), \\
\dot{x}_{4}(t)=2.425 \sin \left(x_{3}(t)\right)+\frac{k r(l-b)}{2 J_{1}} \\
+0.025 \sin \left(x_{2}(t)\right)+u_{2} \\
+\frac{x_{3}\left(t-0.4\left(1+\sin ^{2}(t)\right)\right)}{1+x_{3}^{2}\left(t-0.4\left(1+\sin ^{2}(t)\right)\right)} \\
y_{1}=x_{1}(t) \\
y_{2}=x_{3}(t) .
\end{gathered}
$$

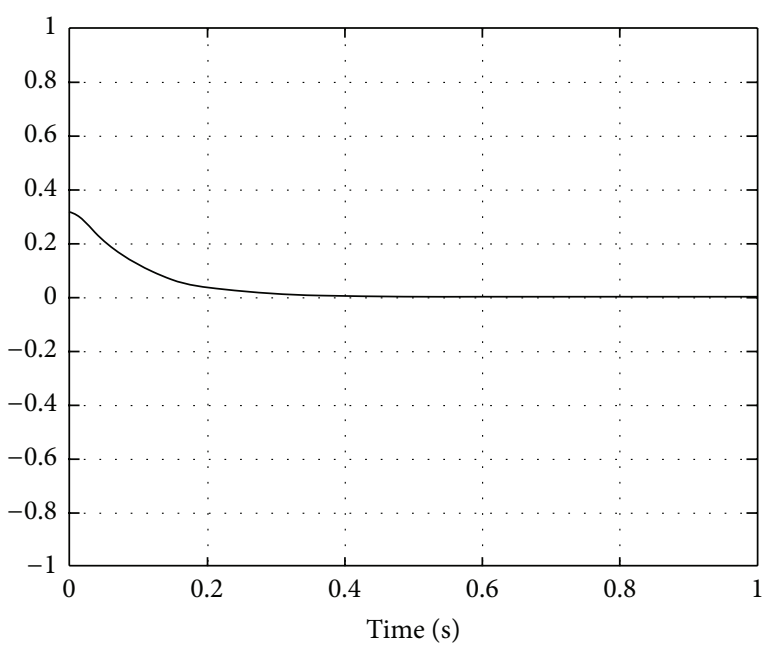

FIgURE 2: The output angular position $x_{1}\left(\theta_{1}\right)$ of the two inverted pendulums.

Now we will show how to explicitly construct a controller that tracks the desired signals $y_{d}^{1}=y_{d}^{2}=0$ and attenuates the disturbance's effect on the output terminal to an arbitrary degree of accuracy. Let us arbitrarily choose $a_{1}^{1}=a_{2}^{1}=a_{1}^{2}=$ $a_{2}^{2}=10$, and the solution of Lyapunov equation is given as $A_{\text {phase }}^{1}=A_{\text {phase }}^{2}=\left[\begin{array}{cc}0 & 1 \\ -10 & -10\end{array}\right], Q^{1}=Q^{2}=\left[\begin{array}{cc}1.05 & 0.05 \\ 0.05 & 0.055\end{array}\right]$, $\lambda_{\text {max }}\left(Q^{1}\right)=\lambda_{\text {max }}\left(Q^{2}\right)=1.0525, \lambda_{\text {min }}\left(Q^{1}\right)=\lambda_{\text {min }}\left(Q^{2}\right)=$ $0.0525, \lambda_{\max }^{*}=1.0525$, and $\lambda_{\text {min }}^{*}=0.0525$. From (31), we obtain the desired tracking controllers

$$
\begin{aligned}
& u_{1}=-1000 x_{1}-100 x_{2}-1.935 \sin x_{1}-0.025 \sin x_{3}, \\
& u_{2}=-1000 x_{3}-100 x_{4}-2.425 \sin x_{3}-0.025 \sin x_{2} .
\end{aligned}
$$

It can be verified that the relative conditions of Theorem 4 are satisfied with $\kappa=0.1, B_{d}^{1}=B_{d}^{2}=0, N_{2}=2.82, k=$ $20 \sqrt{\kappa}$, and $k_{11}=2.82$. Hence the tracking controllers will steer the output tracking errors of the closed-loop system, starting from any initial value to be asymptotically attenuated to zero by virtue of Theorem 4 . The complete trajectories of the outputs are depicted in Figures 2 and 3.

\section{Conclusion}

In this paper, we propose a novel control design which globally solves the almost disturbance decoupling problem for multiinput multioutput nonlinear time delay large-scale system via the fuzzy feedback linearization approach. The investigation of feedback linearization of nonlinear timedelay large-scale control systems by diffeomorphism has been proposed. Moreover, a practical industrial system of two inverted pendulums coupled by a spring demonstrates the applicability of the proposed feedback linearization method. Simulation results exploit the fact that the proposed methodology is successfully utilized to solve the feedback linearization problem and achieve the desired almost disturbance decoupling performance of the overall system. 


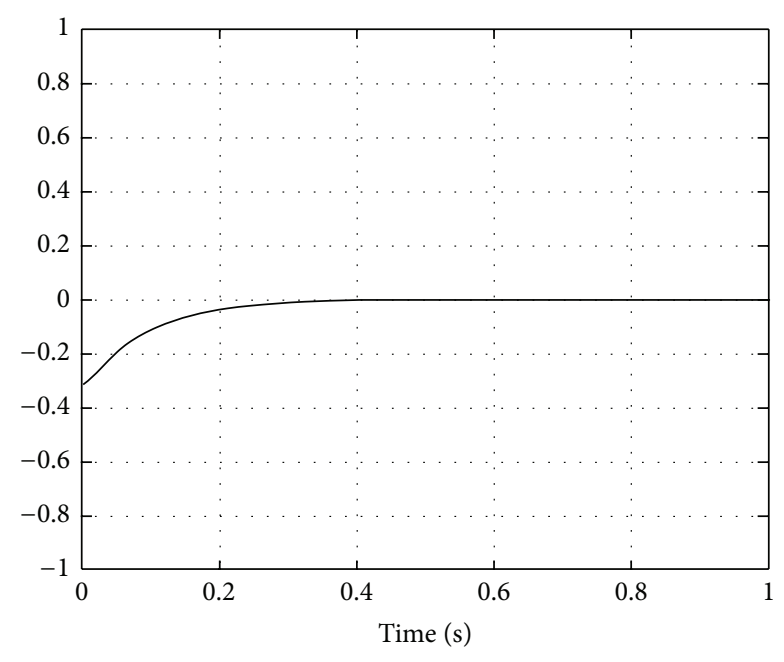

FIgURE 3: The output angular position $x_{3}\left(\theta_{2}\right)$ of the two inverted pendulums.

\section{References}

[1] Q. Zhou, P. Shi, S. Xu, and H. Li, "Adaptive output feedback control for nonlinear time-delay systems by fuzzy approximation approach," IEEE Transactions on Fuzzy Systems, vol. 21, no. 2, pp. 301-313, 2013.

[2] R. D. Driver, Ordinary and Delay Differential Equations, Springer, New York, NY, USA, 1977.

[3] A. H. Gorecki, S. Fuksa, P. Grabowski, and A. Korytowski, Analysis and Synthesis of Time Delay Systems, John Wiley \& Sons, New York, NY, USA, 1989.

[4] H. Trinh and M. Aldeen, "Output tracking for linear uncertain time-delay systems," IEE Proceedings-Control Theory and Applications, vol. 143, no. 6, pp. 481-488, 1996.

[5] S. Phoojaruenchanachai, K. Uahchinkul, and Y. Prempraneerach, "Robust perturbed systems: a geometric approach," International Journal of Control, vol. 145, no. 1, pp. 87-91, 1998.

[6] P. H. A. Ngoc, "Stability of positive differential systems with delay," IEEE Transactions on Automatic Control, vol. 58, no. 1, pp. 203-209, 2013.

[7] W. H. Kwon and A. E. Pearson, "Feedback stabilization of linear systems with delayed control," IEEE Transactions on Automatic Control, vol. 25, no. 2, pp. 266-269, 1980.

[8] C. D. Persis, T. N. Jensen, R. Ortega, and R. Wisniewski, “Output regulation of large-scale hydraulic networks," IEEE Transactions on Control Systems Technology, 2013.

[9] Y. Guo, S. H. Hosseini, C. Y. Tang, J. N. Jiang, and R. G. Ramakumar, "An approximate wind turbine control system model for wind farm power control," IEEE Transactions on Sustainable Energy, vol. 4, no. 1, pp. 262-274, 2013.

[10] D. Yue, E. Tian, and Q.-L. Han, "A delay system method for designing event-triggered controllers of networked control systems," IEEE Transactions on Automatic Control, vol. 58, no. 2, pp. 475-481, 2013.

[11] B. Rahmani and A. H. D. Markazi, "Variable selective control method for networked control systems," IEEE Transactions on Control Systems Technology, vol. 21, no. 3, pp. 975-982, 2013.

[12] H. Gao, T. Chen, and J. Lam, "A new delay system approach to network-based control," Automatica, vol. 44, no. 1, pp. 39-52, 2008.
[13] X. Jiang, Q.-L. Han, S. Liu, and A. Xue, "A new $H_{\infty}$ stabilization criterion for networked control systems," IEEE Transactions on Automatic Control, vol. 53, no. 4, pp. 1025-1032, 2008.

[14] J. Yang, J. Tan, Z. Shu, and L. Chen, "Approximate variable structure control for transient load in large hydraulic press," in Proceedings of the 5th International Conference on Measuring Technology and Mechatronics Automation, pp. 310-312, Hong Kong, January 2013.

[15] N. Nasiri, H. Sadjadian, and A. Mohammad, "Voltage-based control of a flexible-joint electrically driven robot using backstepping approach," in Proceedings of the 4th Power Electronics, Drive Systems and Technologies Conference, pp. 541-546, Tehran, Iran, February 2013.

[16] M. Qin and J. Xu, "Improved pulse regulation control technique for switching DC-DC converters operating in DCM," IEEE Transactions on Industrial Electronics, vol. 60, no. 5, pp. 18191830, 2013.

[17] C. G. Claudel, T. Chamoin, and A. M. Bayen, "Solutions to estimation problems for Scalar Hamilton-Jacobi equations using linear programming," IEEE Transactions on Control Systems Technology, 2013.

[18] A. Isidori, " $H_{\infty}$ control via measurement feedback for affine nonlinear systems," International Journal of Robust and Nonlinear Control, vol. 4, no. 4, pp. 553-574, 1994.

[19] L. Paunonen and S. Pohjolainen, "Reduced order internal models in robust output regulation," IEEE Transactions on Automatic Control, 2013.

[20] S. Gopalswamy and J. K. Hedrick, "Tracking nonlinear nonminimum phase systems using sliding control," International Journal of Control, vol. 57, no. 5, pp. 1141-1158, 1993.

[21] A. Bidram, A. Davoudi, F. L. Lewis, and J. M. Guerrero, "Distributed cooperative secondary control of microgrids using feedback linearization," IEEE Transactions on Power Systems, vol. 28, no. 3, pp. 3462-3470, 2013.

[22] A. Hamadi, S. Rahmani, and K. Al-Haddad, "Digital control of a shunt hybrid power filter adopting a nonlinear control approach," IEEE Transactions on Industrial Informatics, 2013.

[23] Y. H. Liu, N. R. Watson, K. L. Zhou, and B. F. Yang, "converter system nonlinear modelling and control for transmission applications-part II: CSC systems," IEEE Transactions on Power Delivery, vol. 28, no. 3, pp. 1391-1401, 2013.

[24] D. Chwa, "Tracking control of differential-drive wheeled mobile robots using a backstepping-like feedback linearization," IEEE Transactions on Systems, Man, and Cybernetics, Part A, vol. 40, no. 6, pp. 1285-1295, 2010.

[25] J. C. Willems, "Almost invariant subspaces: an approach to high gain feedback design. I. Almost controlled invariant subspaces," IEEE Transactions on Automatic Control, vol. 26, no. 1, pp. 235252, 1981.

[26] R. Marino, W. Respondek, and A. J. van der Schaft, "Almost disturbance decoupling for single-input single-output nonlinear systems," IEEE Transactions on Automatic Control, vol. 34, no. 9, pp. 1013-1017, 1989.

[27] X. Liu and Z. Lin, "Further results on disturbance attenuation for multiple input multiple output nonlinear systems," in Proceedings of the American Control Conference (ACC '10), pp. 6342-6347, Baltimore, Md, USA, July 2010.

[28] Z. Zhong and J. Wang, "Looper-tension almost disturbance decoupling control for hot strip finishing mill based on feedback linearization," IEEE Transactions on Industrial Electronics, vol. 58, no. 8, pp. 3668-3679, 2011. 
[29] S. Kunimatsu, M. Ishitobi, and T. Fujii, "Decentralized PID control for systems with relative degree no more than 2, in Proceedings of the SICE Annual Conference, pp. 1106-1109, Akita, Japan, August 2012.

[30] F. Henrotte, H. Heumann, E. Lange, and K. Hameyer, "Upwind 3-D vector potential formulation for electromagnetic braking simulations," IEEE Transactions on Magnetics, vol. 46, no. 8, pp. 2835-2838, 2010.

[31] H. K. Khali, Nonlinear Systems, Prentice-Hall, Upper Saddle River, NJ, USA, 1996.

[32] R. Marino and P. Tomei, "Nonlinear output feedback tracking with almost disturbance decoupling," IEEE Transactions on Automatic Control, vol. 44, no. 1, pp. 18-28, 1999.

[33] K. Khorasani and P. V. Kokotovic, "A corrective feedback design for nonlinear systems with fast actuators," IEEE Transactions on Automatic Control, vol. 31, no. 1, pp. 67-69, 1986.

[34] R. Marino and P. V. Kokotovic, "A geometric approach to nonlinear singularly perturbed control systems," Automatica, vol. 24, no. 1, pp. 31-41, 1988. 


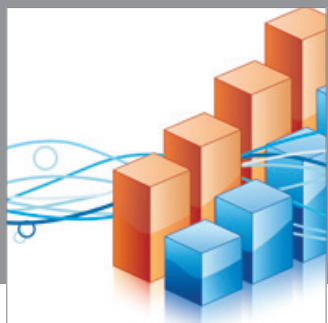

Advances in

Operations Research

mansans

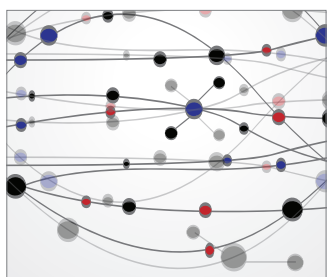

The Scientific World Journal
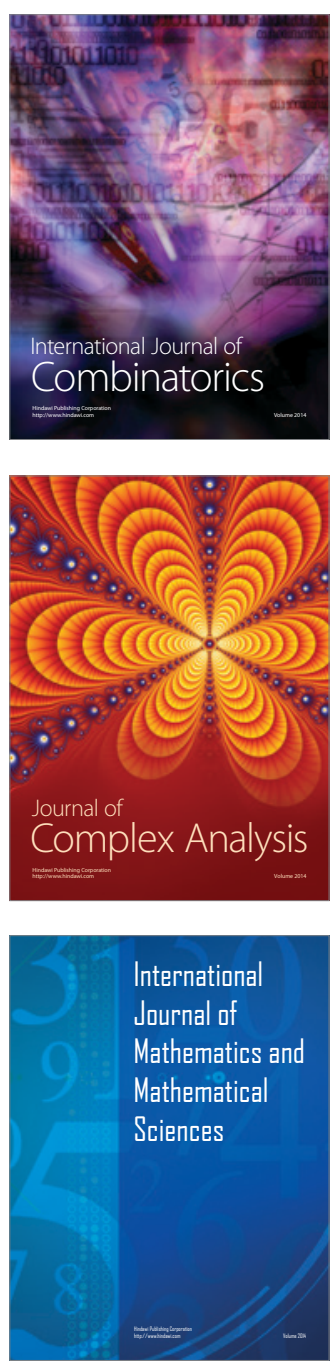
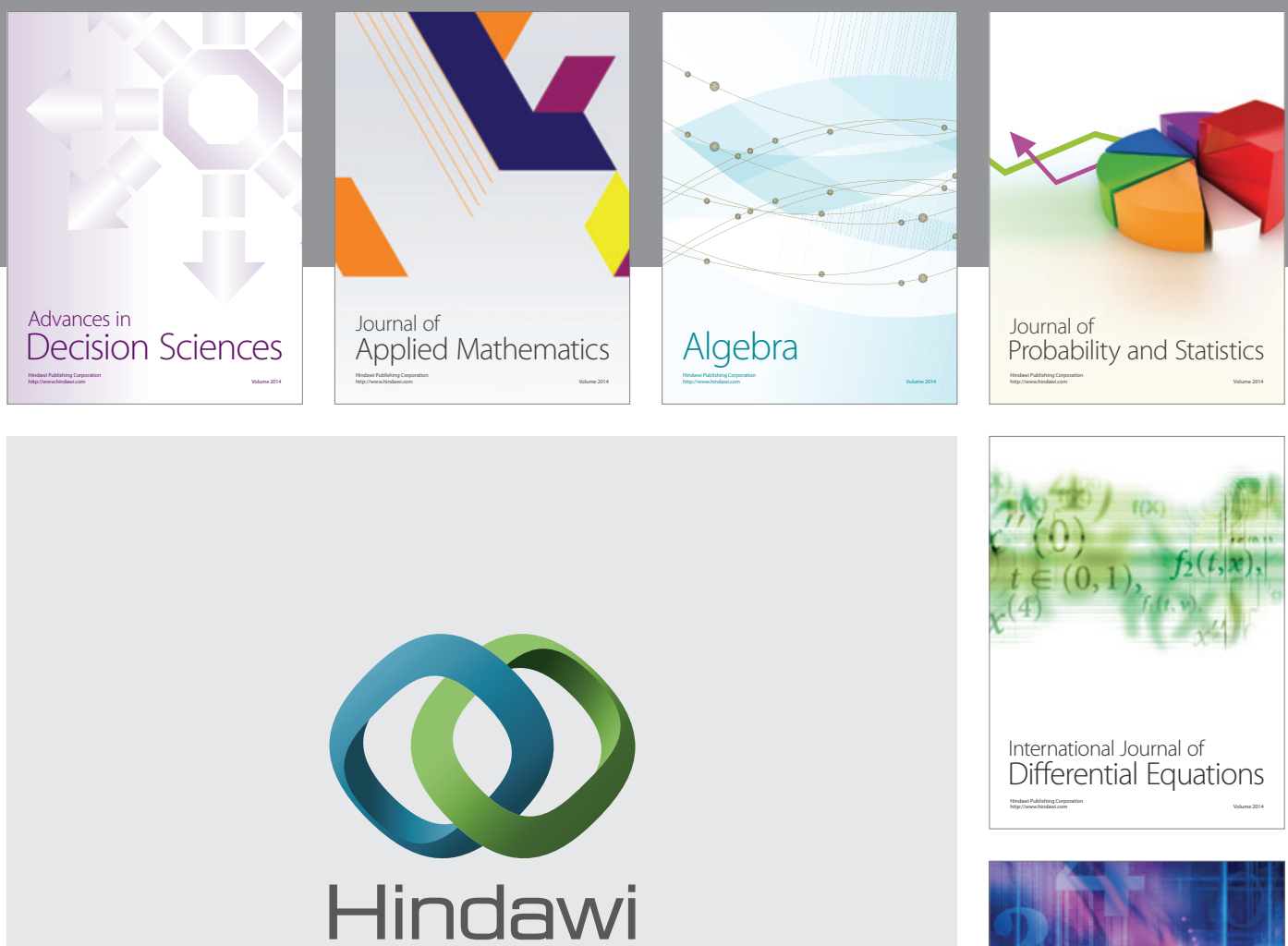

Submit your manuscripts at http://www.hindawi.com
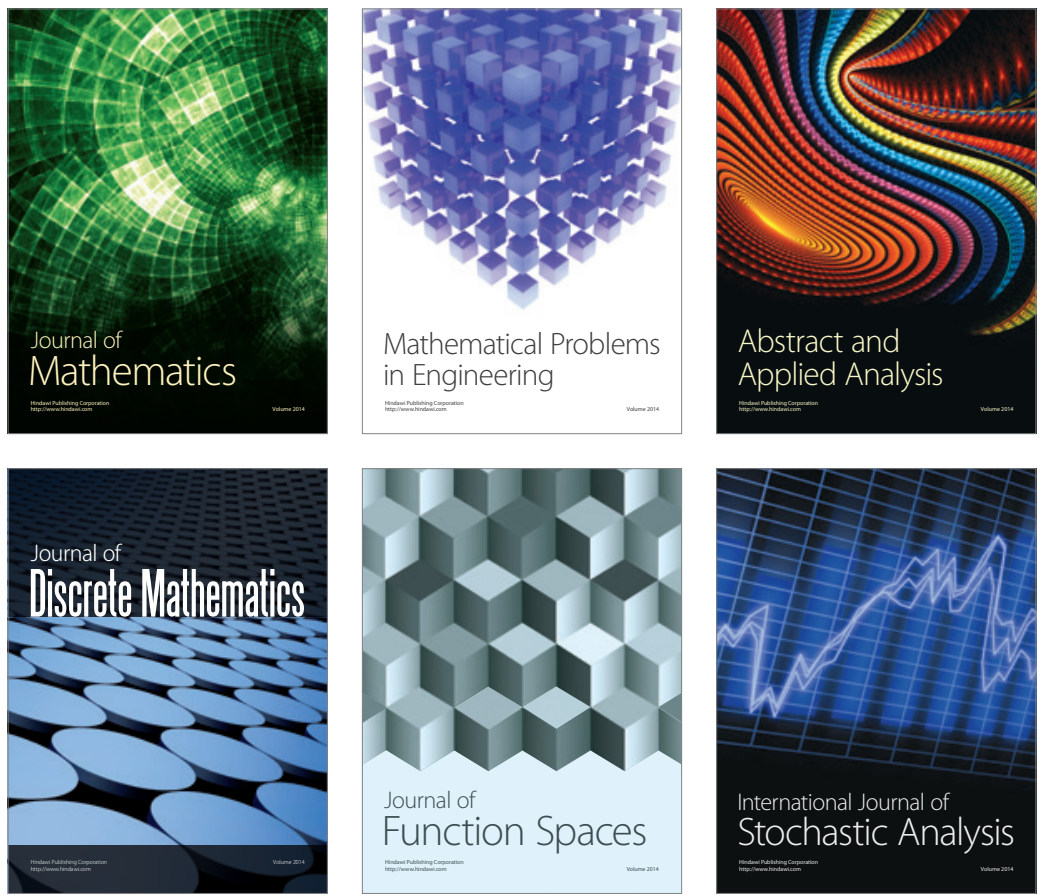

Journal of

Function Spaces

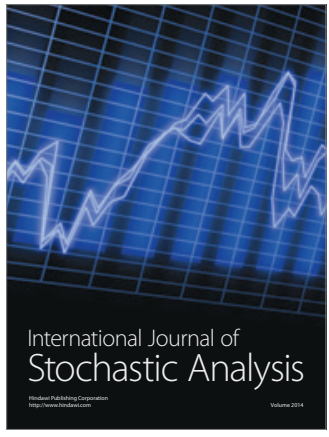

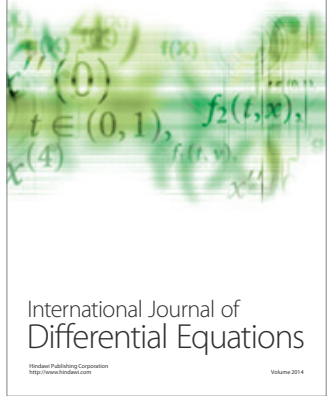
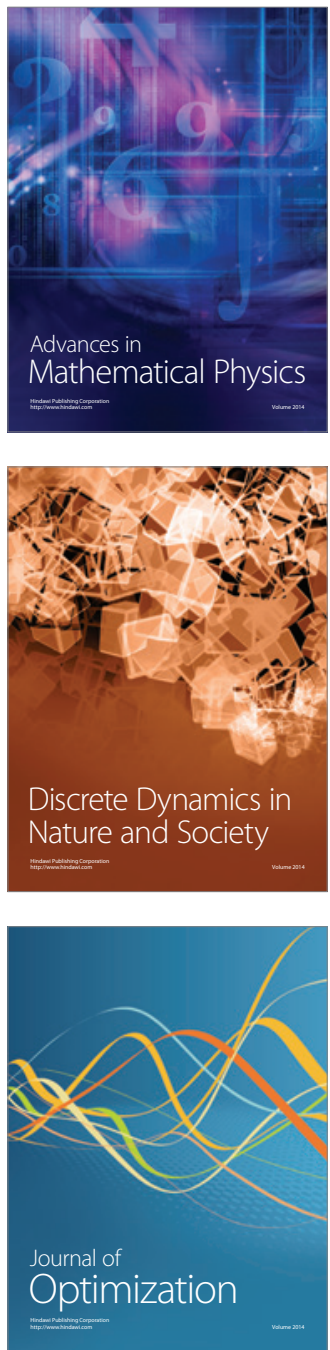